\title{
An axiomatic characterization of a value for games in partition function form
}

\author{
Cheng-Cheng $\mathrm{Hu} \cdot$ Yi-You Yang
}

Received: 26 January 2009/Accepted: 3 July 2009/Published online: 8 January 2010

(C) Spanish Economic Association and Fundación SEPI 2010

\begin{abstract}
An extension of the Shapley value for games in partition function form is proposed in the paper. We introduce a version of the marginal contributions for environments with externalities. The dummy property related to it is defined. We adapt the system of axioms provided by Shapley (A value for $n$-Person games. In: Kuhn H, Tucker A (eds) Contributions to the theory of games II. Princeton University Press, Princeton, pp 307-317, 1953) to characterize our value. In addition, we discuss a relationship between the $\alpha$-Shapley values proposed by Fujinaka (On the marginality principle in partition function form games. Mimeo, Graduate School of Economics, Kobe University, Japan, 2004) and the values constructed through the average approach provided by Macho-Stadler et al. (J Econ Theory 135:339356, 2007).
\end{abstract}

Keywords Externalities · Marginal contributions · Shapley value

JEL Classification C71 $\cdot$ D62

\section{Introduction}

One of the main purposes of game theory is to study how to divide the joint profits among players when they cooperate together. A value for coalitional games is a

The authors are grateful to an anonymous referee whose suggestions improve the paper substantially.

\section{C.-C. Hu $(\bowtie)$}

Department of Finance, Providence University, Taichung 43301, Taiwan e-mail: cchu@pu.edu.tw 
solution which provides an allocation for players' payoffs. Shapley (1953) suggests an axiomatic approach to this issue. In his characterization, the axioms efficiency, symmetry, additivity and the dummy property determine uniquely a value which is called the Shapley value today. The Shapley value can be formulated as the average of players' marginal contributions to all coalitions. Young (1985) shows that the axioms marginality, efficiency and symmetry also yield an axiomatic characterization of the Shapley value. The Shapley value possesses many desirable properties and becomes one of the most famous solutions for characteristic function form games. Nevertheless, the Shapley value cannot give a recommendation for the allocation of payoffs of players in the situation where externalities across coalitions are present.

In the presence of externalities, Thrall and Lucas (1963) introduce games in partition function form as a generalization of characteristic function form games. Recently, some game theorists study extensions of the Shapley value for games in partition function form. For instance, Potter (2000), Fujinaka (2004), Albizuri et al. (2005), Macho-Stadler et al. (2007), Dutta et al. (2008) and McQuillin (2009) are contributions to this line of research. The axiomatic approach is one of methods to justify solution concepts for coalitional games. There are several extensions of the Shapley value for games in partition function form characterized by various adaptations of Shapley's axiomatic system. Myerson (1977) provides an extension of the Shapley value for games in partition function form and a set of axioms which determines uniquely his value. A version of the carrier axiom is proposed and plays a key role in his characterization. Bolger (1989), Pham Do and Norde (2007) and de Clippel and Serrano (2008) also adapt Shapley's axiomatic system to characterize their extensions of the Shapley value for games in partition function form. In the paper, we consider environments with externalities and provide the value $\Phi$ which is an extension of the Shapley value for games in partition function form. We introduce a version of marginal contributions of players to coalitions by which the dummy property is defined. We adapt the system of axioms provided by Shapley (1953) to get a characterization of the value $\Phi$.

Fujinaka (2004) and Macho-Stadler et al. (2007) provide the class of the $\alpha$ Shapley values and the class of values constructed through the "average approach", respectively. Fujinaka introduces the concept of the $\alpha$-marginal contributions constructed as an expected average of the marginal contributions over some partitions. Fujinaka adapts Young's axiomatic system and shows that the axioms $\alpha$ marginality, efficiency and symmetry determine uniquely the $\alpha$-Shapley value. For each partition function form game $v$, Macho-Stadler et al. (2007) perform a weighted average of the partition function $v$ to obtain the game $\tilde{v}$ in characteristic function form. The average approach produces a value $\phi$ for games in partition function form by defining $\phi(v)=\operatorname{Sh}(\tilde{v})$ for all games $v$. We say a value is constructed through the average approach if it can be derived through the procedure described above. It is interesting to note that our value $\Phi$, the value $\varphi^{*}$ proposed by Macho-Stadler et al. (2007) and the externality-free value (de Clippel and Serrano 2008) are in both classes. In particular, they all can be constructed through the average approach with the positive and symmetric weights. Due to the observation, we find a specific subclass of values constructed through the average approach in which each value is an $\alpha$-Shapley value. 
The paper is organized as follows. Section 2 presents definitions and notations. In Sect. 3, we provide an axiomatic result of the value $\Phi$. In Sect. 4, we have a discussion on a relationship between the $\alpha$-Shapley values and the values constructed through the average approach.

\section{Definitions and conventions}

Let $N$ be a non-empty finite set of players. A non-empty subset of $N$ is called a coalition. A partition $\mathcal{P}$ of $N$ is a collection of disjoint subsets of $N$ such that their union is $N$ and $\emptyset \in \mathcal{P}$. Elements of a partition are called atoms. Let $\Gamma$ denote the collection of all partitions of $N$. A permutation $\pi$ of $N$ is a one-to-one function from $N$ onto itself. Denote $\Pi$ to be the set of all permutations of $N$. An embedded coalition is a pair $(S, \mathcal{P})$ where $\mathcal{P}$ is a partition of $N$ and $S$ is an atom of $\mathcal{P}$. The embedded coalition $(S, \mathcal{P})$ is called non-trivial if $S$ is non-empty. The collection of all non-trivial embedded coalitions is denoted by $E C$.

A game in partition function form, or simply a game, is a function $v$ that assigns to each embedded coalition $(S, \mathcal{P})$ a real number $v(S, \mathcal{P})$. We assume that $v(\emptyset, \mathcal{P})=$ 0 for all $\mathcal{P} \in \Gamma \cdot v(S, \mathcal{P})$ is called the worth of the coalition $S$ with respect to the partition $\mathcal{P}$. A game is with externalities if and only if the worth of some coalition depends on the way the other players are organized. That is, there exists a coalition $S$ and two embedded coalitions $(S, \mathcal{P}),\left(S, \mathcal{P}^{\prime}\right)$ such that $v(S, \mathcal{P}) \neq v\left(S, \mathcal{P}^{\prime}\right)$. A game is with no externalities if and only if, for each coalition $S$, the worth of $S$ is independent of the way the other players are organized. That is, $v(S, \mathcal{P})=v\left(S, \mathcal{P}^{\prime}\right)$ for each pair of embedded coalitions $(S, \mathcal{P})$ and $\left(S, \mathcal{P}^{\prime}\right)$. In the latter case, a partition function is called a characteristic function.

A value $\phi$ is a function that assigns to each game $v$ a vector $\phi(v)$ in $\mathbb{R}^{N}$. We say that a value $\phi$ satisfies efficiency if $\sum_{i \in N} \phi_{i}(v)=v(N,\{N, \emptyset\})$ for all games $v$. The efficiency property says that the allocation selected by the value $\phi$ is feasible and the resource of players is not wasted.

Let $\pi \in \Pi$. For each coalition $S \subseteq N$, let $\pi S=\{\pi(i): i \in S\}$. For each partition $\mathcal{P}$, let $\pi \mathcal{P}=\{\pi S: S \in \mathcal{P}\}$. Given two members $i$ and $j$ of $N$, let $\pi^{i j} \in \Pi$ such that $\pi^{i j}(i)=j, \pi^{i j}(j)=i$ and $\pi(k)=k$ for all $k \in N \backslash\{i, j\}$. Players $i$ and $j$ are symmetric in $v$ if $v(S, \mathcal{P})=v\left(\pi^{i j} S, \pi^{i j} \mathcal{P}\right)$ for each $(S, \mathcal{P})$. We say that a value $\phi$ satisfies symmetry if players $i$ and $j$ are symmetric in $v$ then $\phi_{i}(v)=\phi_{j}(v)$. The symmetry axiom says that if two players are substituted in the game, they should get the same payoff.

We say that a value $\phi$ satisfies additivity if $\phi(u+v)=\phi(u)+\phi(v)$ for all games $u$ and $v$. When players distribute their payoffs on two different issues, the additivity property says that it makes no difference for players to consider the two issues together or one by one.

Let $S \subseteq N$ and let $i \in N$. For convenience, we will write $S \backslash i$ instead of $S \backslash\{i\}$. Let $\mathcal{O} \in \Gamma$. Denote $\mathcal{O}_{S}=\{T \backslash S: T \in \mathcal{O}\} \cup\{S\}$. Given $(S, \mathcal{P}) \in E C$, let

$$
\Gamma_{S, \mathcal{P}}=\left\{\mathcal{O} \in \Gamma: \mathcal{O}_{S}=\mathcal{P}\right\}
$$

Let $\mathcal{P} \in \Gamma$ and let $\mathcal{P}(i)$ denote the member of $\mathcal{P}$ to which player $i$ belongs. Let $v$ be a game and let $\mathcal{O} \in \Gamma_{\mathcal{P}(i), \mathcal{P}}$. The $\mathcal{O}$-marginal contribution of player $i$ to $\mathcal{P}$ is defined by 


$$
v(\mathcal{P}(i), \mathcal{P})-v\left(\mathcal{P}(i) \backslash i, \mathcal{O}_{\mathcal{P}(i) \backslash i}\right) .
$$

It is quite common that some persons leave their original organizations, respectively, and establish their own coalition. Assume that such deviation results in the generation of partition $\mathcal{P}$. We also assume that partition $\mathcal{O}$ is the original coalition structure before the deviation of coalition $\mathcal{P}(i)$. Then the $\mathcal{O}$-marginal contribution of player $i$ to $\mathcal{P}$ presents player $i$ 's contribution to coalition $\mathcal{P}(i)$ when he is the last person to join the deviation. The set $\Gamma_{\mathcal{P}(i), \mathcal{P}}$ is the collection of all possible partitions $\mathcal{O}$ from which coalition $\mathcal{P}(i)$ can deviate to form partition $\mathcal{P}$. Therefore, player $i$ may have different $\mathcal{O}$-marginal contributions. If we adopt the concept of the $\mathcal{O}$-marginal contributions to measure the bargaining power of player $i$, how should we evaluate his bargaining ability according to his $\mathcal{O}$-marginal contributions? The following approach is one of the possible ways to measure player $i$ 's bargaining ability.

Player $i$ is called a weak dummy in the game $v$ if

$$
v(\mathcal{P}(i), \mathcal{P})-v\left(\mathcal{P}(i) \backslash i, \mathcal{O}_{\mathcal{P}(i) \backslash i}\right)=0,
$$

for all partitions $\mathcal{P}$ of $N$ and for all $\mathcal{O} \in \Gamma_{\mathcal{P}(i), \mathcal{P}}$. We say that a value $\phi$ satisfies the weak dummy property (Bolger 1989) if player $i$ is a weak dummy in $v$, then $\phi_{i}(v)=0$. That is, if for each partition $\mathcal{P}$ and each $\mathcal{O} \in \Gamma_{\mathcal{P}(i), \mathcal{P}}$, the $\mathcal{O}$-marginal contribution of player $i$ to $\mathcal{P}$ is 0 , then we view that player $i$ has no bargaining power and hence his payoff should be zero. Note that Eq. (1) is equivalent to the following

$$
v(\mathcal{P}(i), \mathcal{P})-v\left(\mathcal{P}(i) \backslash i, \mathcal{P}^{\prime}\right)=0
$$

for all partitions $\mathcal{P}$ of $N$, where $\mathcal{P}^{\prime}$ is any partition of $N$ obtained from $\mathcal{P}$ by moving player $i$ to some other coalition in $\mathcal{P}$.

Nevertheless, one may prefer an aggregate index to segregate ones in some situations. For instance, in order to identify whether an applicant meets the eligibility requirements of financial aid, he is required to report his total income instead of all his individual earnings. In a problem of measuring the effect of an investment plan which including many projects, one may prefer knowing the total profit of all projects to identifying the consequence of each project. Different to Bolger's separate measure, we adopt the following approach to evaluate the bargaining ability of a player.

The marginal contribution of player $i$ to the partition $\mathcal{P}$ is given by

$$
v^{i}(\mathcal{P})=\sum_{\mathcal{O} \in \Gamma_{\mathcal{P}(i), \mathcal{P}}} v(\mathcal{P}(i), \mathcal{P})-v\left(\mathcal{P}(i) \backslash i, \mathcal{O}_{\mathcal{P}(i) \backslash i}\right) .
$$

The amount $v^{i}(\mathcal{P})$ which is the sum of all $\mathcal{O}$-marginal contributions of player $i$ to $\mathcal{P}$ can be viewed as an aggregate index to measure player $i$ 's bargaining power. If $v^{i}(\mathcal{P})=0$ for all $\mathcal{P}$, it means player $i$ has no bargaining power and his payoff should be zero. We define the property formally in the following.

Let $v$ be a game and let $i \in N$. Player $i$ is called a dummy in $v$ if $v^{i}(\mathcal{P})=0$ for all partitions $\mathcal{P}$. We say that a value $\phi$ satisfies the dummy property if for any 
player $i$ which is a dummy player in the game $v, \phi_{i}(v)=0$. The dummy property describes that a player gets nothing if his marginal contribution to each partition is zero.

Next, we introduce the value $\Phi$. Let $v$ be a game and let $\pi \in \Pi$. We assume that $N=\{1,2, \ldots, n\}$. Denote $A_{k}=\pi\{1,2, \ldots, k\}$ for all $1 \leq k \leq n$ and $A_{0}=\emptyset$. For each $\mathcal{O} \in \Gamma$, let $z^{\mathcal{O}, \pi}=\left(z_{i}^{\mathcal{O}, \pi}\right)_{i \in N}$ where

$$
z_{\pi(k)}^{\mathcal{O}, \pi}=v\left(A_{k}, \mathcal{O}_{A_{k}}\right)-v\left(A_{k-1}, \mathcal{O}_{A_{k-1}}\right),
$$

for all $k \in N$. Let $D$ be a set and let $|D|$ be the cardinality of $D$. Let

$$
z^{\pi}=\frac{1}{|\Gamma|} \sum_{\mathcal{O} \in \Gamma} z^{\mathcal{O}, \pi}
$$

$\Phi(v)$ is given by

$$
\Phi(v)=\frac{1}{|N| !} \sum_{\pi \in \Pi} z^{\pi} .
$$

Let $k \in N$ and let $\mathbf{K}=\left\{\mathcal{P} \in \Gamma: \mathcal{P} \ni A_{k}\right\}$. It follows that

$$
\Gamma=\bigcup_{\mathcal{P} \in \mathbf{K}} \Gamma_{A_{k}, \mathcal{P}}
$$

Note that $\Gamma_{A_{k}, \mathcal{P}} \cap \Gamma_{A_{k}, \mathcal{P}^{\prime}}=\emptyset$ for all $\mathcal{P}, \mathcal{P}^{\prime} \in \mathbf{K}$ with $\mathcal{P} \neq \mathcal{P}^{\prime}$. It holds that

$$
\begin{aligned}
z_{\pi(k)}^{\pi} & =\frac{1}{|\Gamma|} \sum_{\mathcal{O} \in \Gamma}\left\{v\left(A_{k}, \mathcal{O}_{A_{k}}\right)-v\left(A_{k-1}, \mathcal{O}_{A_{k-1}}\right)\right\} \\
& =\frac{1}{|\Gamma|} \sum_{\mathcal{P} \in \mathbf{K} \mathcal{O} \in \Gamma_{A_{k}, \mathcal{P}}}\left\{v\left(A_{k}, \mathcal{O}_{A_{k}}\right)-v\left(A_{k-1}, \mathcal{O}_{A_{k-1}}\right)\right\} \\
& =\frac{1}{|\Gamma|} \sum_{\mathcal{P} \in \Gamma, \mathcal{P} \ni A_{k}}\left\{\sum_{\mathcal{O} \in \Gamma_{A_{k}}, \mathcal{P}} v\left(A_{k}, \mathcal{P}\right)-v\left(A_{k-1}, \mathcal{O}_{A_{k-1}}\right)\right\} \\
& =\frac{1}{|\Gamma|} \sum_{\mathcal{P} \in \Gamma, \mathcal{P} \ni A_{k}} v^{\pi(k)}(\mathcal{P}) .
\end{aligned}
$$

By (3) and (4), $\Phi_{i}(v)$ also can be expressed by

$$
\Phi_{i}(v)=\sum_{\substack{S \subseteq N, S \ni i \\ \mathcal{O} \in \Gamma}} \frac{(|S \backslash i| !)(|N \backslash S| !)}{|N| !|\Gamma|}\left[v\left(S, \mathcal{O}_{S}\right)-v\left(S \backslash i, \mathcal{O}_{S \backslash i}\right)\right]
$$

for all $i \in N$. The Shapley value $S h$ is a function that assigns to each characteristic function form game $v$ a vector $\operatorname{Sh}(v)$ in $\mathbb{R}^{N}$. The ith coordinate of $\operatorname{Sh}(v)$ is given by

$$
S h_{i}(v)=\sum_{S \subseteq N, S \ni i} \frac{(|S \backslash i| !)(|N \backslash S| !)}{|N| !}(v(S)-v(S \backslash i)) .
$$




\section{Axiomatic characterization of the value $\Phi$}

In the following, we provide an axiomatic characterization of the value $\Phi$. First, we introduce the unanimity games to help us to characterize the value $\Phi$. For every nontrivial embedded coalition $(T, \mathcal{R})$, the game $v^{T, \mathcal{R}}$ is defined by

$$
v^{T, \mathcal{R}}(S, \mathcal{P})=\left\{\begin{array}{cc}
\frac{1}{\mid \Gamma_{S, \mathcal{P}},}, & \text { if } S \supseteq T \text { and } \mathcal{P}=\mathcal{R}_{S}, \\
0, & \text { otherwise, }
\end{array}\right.
$$

for all non-trivial embedded coalitions $(S, \mathcal{P})$ The game $v^{T, \mathcal{R}}$ is called the unanimity game with respect to $(T, \mathcal{R})$.

Lemma 1 The collection of all the unanimity games is a basis of the space of games in partition function form.

Proof Let $\Theta$ denote the collection of all the unanimity games. That is

$$
\Theta=\left\{v^{T, \mathcal{R}}:(T, \mathcal{R}) \in E C\right\} .
$$

We will show that $\Theta$ is linearly independent. If not, then there exists a collection of coefficients $\beta^{T, \mathcal{R}}$, not all zero, such that $\sum_{(T, \mathcal{R}) \in E C} \beta^{T, \mathcal{R}} v^{T, \mathcal{R}}(S, \mathcal{P})=0$ for all $(S, \mathcal{P}) \in E C$. There exists an embedded coalition $\left(T_{0}, \mathcal{R}_{0}\right)$ such that $\beta^{T_{0}, \mathcal{R}_{0}} \neq 0$ and $\beta^{T, \mathcal{R}}=0$ for all $(T, \mathcal{R}) \in E C, T \subset T_{0}$ and $T \neq T_{0}$. Then

$$
\begin{aligned}
\sum_{(T, \mathcal{R}) \in E C} \beta^{T, \mathcal{R}} v^{T, \mathcal{R}}\left(T_{0}, \mathcal{R}_{0}\right) & =\sum_{(T, \mathcal{R}) \in E C, T \subseteq T_{0}} \beta^{T, \mathcal{R}} v^{T, \mathcal{R}}\left(T_{0}, \mathcal{R}_{0}\right) \\
& =\beta^{T_{0}, \mathcal{R}_{0}} v^{T_{0}, \mathcal{R}_{0}}\left(T_{0}, \mathcal{R}_{0}\right) \neq 0 .
\end{aligned}
$$

We obtain a desired contradiction. Since the number $|\Theta|$ is the same as the dimension of the space of games in partition function form, the proof is complete.

The following Lemmas 2 and 3 play the key role for deriving Theorem 4.

Lemma 2 Let $(T, \mathcal{R})$ be a non-trivial embedded coalition. Then every player in $N \backslash T$ is a dummy in the unanimity game $v^{T, \mathcal{R}}$.

Proof Let $i \in N \backslash T$ and let $\mathcal{P}$ be a partition. We will show the following to obtain the desired result.

$$
\left(v^{T, \mathcal{R}}\right)^{i}(\mathcal{P})=\sum_{\mathcal{O} \in \Gamma_{\mathcal{P}(i), \mathcal{P}}}\left[v^{T, \mathcal{R}}(\mathcal{P}(i), \mathcal{P})-v^{T, \mathcal{R}}\left(\mathcal{P}(i) \backslash i, \mathcal{O}_{\mathcal{P}(i) \backslash i}\right)\right]=0 .
$$

There are three cases to be considered.

Case $1 \quad T \nsubseteq \mathcal{P}(i)$. By the assumption $T \nsubseteq \mathcal{P}(i)$, it holds that $T \nsubseteq \mathcal{P}(i) \backslash i$ and

$$
v^{T, \mathcal{R}}(\mathcal{P}(i), \mathcal{P})=0
$$

and

$$
v^{T, \mathcal{R}}\left(\mathcal{P}(i) \backslash i, \mathcal{O}_{\mathcal{P}(i) \backslash i}\right)=0
$$

for all $\mathcal{O} \in \Gamma_{\mathcal{P}(i), \mathcal{P}}$. Then we derive that (5) holds. 
Case $2 \quad T \subseteq \mathcal{P}(i)$ and $\mathcal{P} \neq \mathcal{R}_{\mathcal{P}(i)}$. Clearly, we have (6) holds. Let $\mathcal{O} \in \Gamma_{\mathcal{P}(i), \mathcal{P}}$. Since $\mathcal{O}_{\mathcal{P}(i)}=\mathcal{P} \neq \mathcal{R}_{\mathcal{P}(i)}$, we derive that $\mathcal{O}_{\mathcal{P}(i) \backslash i} \neq \mathcal{R}_{\mathcal{P}(i) \backslash i}$ and (7) holds. We obtain the desired result that $\left(v^{T, \mathcal{R}}\right)^{i}(\mathcal{P})=0$.

Case $3 \quad T \subseteq \mathcal{P}(i)$ and $\mathcal{P}=\mathcal{R}_{\mathcal{P}(i)}$. Since $i \notin T$, we have that $T \subseteq \mathcal{P}(i) \backslash i$. Let

$$
H=\left\{\mathcal{O} \in \Gamma_{\mathcal{P}(i), \mathcal{P}}: \mathcal{O}_{\mathcal{P}(i) \backslash i}=\mathcal{R}_{\mathcal{P}(i) \backslash i}\right\}
$$

It holds that

$$
\begin{aligned}
H & =\left\{\mathcal{O} \in \Gamma: \mathcal{O}_{\mathcal{P}(i) \backslash i}=\mathcal{R}_{\mathcal{P}(i) \backslash i}\right\} \\
& =\Gamma_{\mathcal{P}(i) \backslash i, \mathcal{R}_{\mathcal{P}(i) \backslash i}},
\end{aligned}
$$

and $v^{T, \mathcal{R}}\left(\mathcal{P}(i) \backslash i, \mathcal{O}_{\mathcal{P}(i) \backslash i}\right)=0$ for all $\mathcal{O} \in \Gamma_{\mathcal{P}(i), \mathcal{P}} \backslash H$. It follows that

$$
\begin{aligned}
& \sum_{\mathcal{O} \in \Gamma_{\mathcal{P}(i), \mathcal{P}}} v^{T, \mathcal{R}}\left(\mathcal{P}(i) \backslash i, \mathcal{O}_{\mathcal{P}(i) \backslash i}\right) \\
= & \sum_{\mathcal{O} \in H} v^{T, \mathcal{R}}\left(\mathcal{P}(i) \backslash i, \mathcal{O}_{\mathcal{P}(i) \backslash i}\right)+\sum_{\mathcal{O} \in \Gamma_{\mathcal{P}(i), \mathcal{P}} \backslash H} v^{T, \mathcal{R}}\left(\mathcal{P}(i) \backslash i, \mathcal{O}_{\mathcal{P}(i) \backslash i}\right) \\
= & \left|\Gamma_{\mathcal{P}(i) \backslash i, \mathcal{R}_{\mathcal{P}(i) \backslash i}}\right| v^{T, \mathcal{R}}\left(\mathcal{P}(i) \backslash i, \mathcal{R}_{\mathcal{P}(i) \backslash i}\right) \\
= & 1 .
\end{aligned}
$$

Note that

$$
\sum_{\mathcal{O} \in \Gamma_{\mathcal{P}(i), \mathcal{P}}} v^{T, \mathcal{R}}\left(\mathcal{P}(i), \mathcal{O}_{\mathcal{P}(i)}\right)=\left|\Gamma_{\mathcal{P}(i), \mathcal{P}}\right| v^{T, \mathcal{R}}(\mathcal{P}(i), \mathcal{P})=1
$$

Then (5) holds. By Cases 1, 2 and 3, the proof is complete.

Due to the above Lemma, we will show the following: if a value satisfies efficiency, symmetry and the dummy property, then we can derive players' payoffs allocated by it for all unanimity games.

Lemma 3 Let $\Psi$ be a value satisfying efficiency, symmetry and the dummy property. Let $(T, \mathcal{R})$ be a non-trivial embedded coalition and $\beta \in \mathbb{R}$. Then

$$
\Psi_{i}\left(\beta v^{T, \mathcal{R}}\right)=\left\{\begin{array}{cc}
\frac{\beta}{|T||\Gamma|}, & \text { if } i \in T, \\
0, & \text { otherwise, }
\end{array}\right.
$$

for each $i \in N$.

Proof Let $i \in N$. If $i \notin T$, player $i$ is a dummy in $v^{T, \mathcal{R}}$ by Lemma 2. By the dummy property, we have $\Psi_{i}\left(\beta v^{T, \mathcal{R}}\right)=0$. By efficiency and symmetry, we have that

$$
\begin{aligned}
\Psi_{i}\left(\beta v^{T, \mathcal{R}}\right) & =\frac{1}{|T|}\left\{\beta v^{T, \mathcal{R}}(N,\{N, \emptyset\})-\sum_{j \in N \backslash T} \Psi_{j}\left(\beta v^{T, \mathcal{R}}\right)\right\} \\
& =\frac{\beta}{|T||\Gamma|}
\end{aligned}
$$

for $i \in T$. 
Next, we will show that efficiency, symmetry, additivity, and the dummy property determine uniquely the value $\Phi$. It is known that these axioms are logically independent in Shapley's (1953) axiomatization of the Shapley value for games in characteristic function form.

Theorem $4 \Phi$ is the unique value satisfying efficiency, symmetry, additivity, and the dummy property.

Proof It is clear that the value $\Phi$ satisfies additivity and symmetry. By (4), we derive that $\Phi$ satisfies the dummy property. Next, we show that $\Phi$ satisfies efficiency. Let $v$ be a game. Let $\pi \in \Pi$. Since $\sum_{i \in N} z_{i}^{\mathcal{O}, \pi}=v(N,\{N, \emptyset\})$ for all $\mathcal{O} \in \Gamma$, we derive that $\sum_{i \in N} \Phi_{i}(v)=v(N,\{N, \emptyset\})$ by (2) and (3).

Next, we show the uniqueness part. Let $\Psi$ be a value satisfying efficiency, symmetry, additivity, and the dummy property. Let $v$ be a game. By Lemma 3 , it holds that

$$
\Phi\left(\beta v^{T, \mathcal{R}}\right)=\Psi\left(\beta v^{T, \mathcal{R}}\right)
$$

for every non-trivial embedded coalition $(T, \mathcal{R})$ and $\beta \in \mathbb{R}$.

By Lemma 1, there exists a unique collection of coefficients $\beta^{T, \mathcal{R}}$ such that $v=\sum_{(T, \mathcal{R}) \in E C} \beta^{T, \mathcal{R}} v^{T, \mathcal{R}}$. Since both values $\Phi$ and $\Psi$ satisfy additivity, we derive that

$$
\Phi(v)=\sum_{(T, \mathcal{R}) \in E C} \Phi\left(\beta^{T, \mathcal{R}} v^{T, \mathcal{R}}\right)=\sum_{(T, \mathcal{R}) \in E C} \Psi\left(\beta^{T, \mathcal{R}} v^{T, \mathcal{R}}\right)=\Psi(v) .
$$

The proof is complete.

Bolger (1989) proposes the Bolger value which is an extension of the Shapley value for games in partition function form and the weak dummy property. Since both the Bolger value and our value satisfy efficiency, symmetry, additivity, and the weak dummy property, the dummy property in our characterization cannot be replaced by the weak version.

Let $v$ be a game and let $i \in N$. Player $i$ 's intrinsic marginal contribution to partition $\mathcal{P}$ is given by

$$
v(\mathcal{P}(i), \mathcal{P})-v\left(\mathcal{P}(i) \backslash i, \mathcal{P}_{i}\right)
$$

where $\mathcal{P}_{i}=\{i\} \cup\{T \backslash i: T \in \mathcal{P}\}$. The externality-free value (de Clippel and Serrano 2008) which is an extension of the Shapley value for games in partition function form can be characterized by efficiency, symmetry, additivity, and a version of the dummy property related to the intrinsic marginal contribution. That is, the externality-free value and our value can be characterized by the adaptations of Shapley's characterization. The only difference is the notion of the marginal contribution employed to define the relative dummy property. One may ask the question : Do both adaptations of Shapley's axiomatic system characterize the same value? The answer is negative. The following modification of Example 7 in de Clippel and Serrano (2008) illustrates this fact.

Example 5 Let $v$ be game with the player set $N=\{1,2,3\}$, where 


$$
\begin{aligned}
v(N,\{N, \emptyset\}) & =v(\{2,3\},\{\{1\},\{2,3\}, \emptyset\}) \\
& =v(\{2\},\{\{1\},\{2\},\{3\}, \emptyset\}) \\
& =v(\{3\},\{\{1\},\{2\},\{3\}, \emptyset\}) \\
& =1,
\end{aligned}
$$

and

$$
v(\{1,2\},\{\{1,2\},\{3\}, \emptyset\})=v(\{1,3\},\{\{1,3\},\{2\}, \emptyset\})=0.5,
$$

and $v(S, \mathcal{P})=0$ otherwise. Then $\Phi(v)=\left(\frac{-2}{60}, \frac{31}{60}, \frac{31}{60}\right)$ and the externality-free value of the game $v$ is $\left(\frac{-1}{6}, \frac{7}{12}, \frac{7}{12}\right)$.

\section{The $\alpha$-Shapley values and the average approach}

Fujinaka (2004) proposes the notion of the $\alpha$-marginal contribution and introduces the $\alpha$-Shapley values which are extensions of the Shapley value for games in partition function form. The $\alpha$-marginal contribution is a weighted average of the marginal contributions over different partitions. Let $(S, \mathcal{P})$ be an embedded coalition and let $i \in N$. Let $\gamma_{i}(S, \mathcal{P})$ be the partition obtained by moving player $i$ to coalition $S$. That is,

$$
\gamma_{i}(S, \mathcal{P})=\{S \cup\{i\}\} \cup\{T \backslash i: T \in \mathcal{P}, T \neq S\} .
$$

Let $i \in N$ and let $\mathbb{E}$ be the collection of all embedded coalitions and $\mathbb{E}_{i}=$ $\{(\mathcal{P}(i), \mathcal{P}): \mathcal{P} \in \Gamma\}$. Let $\alpha_{i}$ be a function from $\mathbb{E} \backslash \mathbb{E}_{i}$ to $\mathbb{R}_{+}$and let $\alpha=\left(\alpha_{i}\right)_{i \in N}$. We assume that $\alpha$ satisfies the following conditions.

(1) For each $i \in N$ and $\mathcal{P} \in \Gamma$,

$$
\sum_{S \in \mathcal{P}, S \neq \mathcal{P}(i)} \alpha_{i}\left(\mathcal{P}(i) \backslash i, \gamma_{i}(S, \mathcal{P})\right)=1 .
$$

(2) For each $i \in N,(S, \mathcal{P}) \in \mathbb{E} \backslash \mathbb{E}_{i}$ and $\pi \in \Pi$,

$$
\alpha_{i}(S, \mathcal{P})=\alpha_{\pi(i)}(\pi S, \pi \mathcal{P}) .
$$

Let $v$ be a game. Player $i$ 's $\alpha$-marginal contribution to partition $\mathcal{P}$ is defined by

$$
v_{\alpha}^{i}(\mathcal{P})=\sum_{S \in \mathcal{P}, S \neq \mathcal{P}(i)} \alpha_{i}\left(\mathcal{P}(i) \backslash i, \gamma_{i}(S, \mathcal{P})\right)\left[v(\mathcal{P}(i), \mathcal{P})-v\left(\mathcal{P}(i) \backslash i, \gamma_{i}(S, \mathcal{P})\right)\right] .
$$

Fujinaka (2004) proposes the $\alpha$-Shapley value $\Phi^{\alpha}$ through the concept of the $\alpha$ marginal contribution and uses the above assumptions (1) and (2) to show that $\Phi^{\alpha}$ satisfies efficiency and symmetry.

It is quite interesting to trace back the original states before player $i$ 's deviation resulting in the generation of partition $\mathcal{P}$. For each $S \in \mathcal{P}, S \neq \mathcal{P}(i), \gamma_{i}(S, \mathcal{P})$ is one of the possible partitions from which player $i$ can deviate individually to form partition $\mathcal{P}$. There are various marginal contributions $v(\mathcal{P}(i), \mathcal{P})-$ $v\left(\mathcal{P}(i) \backslash i, \gamma_{i}(S, \mathcal{P})\right)$ of player $i$ induced by his deviations. Each of them can be 
viewed as player $i$ 's bargaining ability in different situations. The $\alpha$-marginal contribution of player $i$ to partition $\mathcal{P}$ can be interpreted an index of player $i$ 's bargaining power by averaging his marginal contributions over different partitions with the weights $\alpha_{i}\left(\mathcal{P}(i) \backslash i, \gamma_{i}(S, \mathcal{P})\right)$. The weights can be viewed as measures of importance. For instance, in a problem of allocating investment costs, one may distribute his capital among all his investment projects with corresponding weights.

We say that a value $\phi$ satisfies $\alpha$-marginality if the following condition is satisfied: Given the two games $u$ and $v$ and $i \in N$, if $u_{\alpha}^{i}(\mathcal{P})=v_{\alpha}^{i}(\mathcal{P})$ for all partitions $\mathcal{P}$ then $\phi_{i}(u)=\phi_{i}(v)$. The axiom $\alpha$-marginality says that, if a player's $\alpha$-marginal contribution to each partition is the same in two games, then the value should pay the same in both games. Fujinaka obtains that the $\alpha$-marginality axiom together with the axioms efficiency and symmetry characterizes the $\alpha$-Shapley value.

Different $\alpha$-marginalities lead to different $\alpha$-Shapley values. There is a link between the $\alpha$-Shapley values and several values proposed in literature. The externality-free value, the Bolger value, the value $\Phi$ and the value $\varphi^{*}$ introduced by Macho-Stadler et al. (2007) are $\alpha$-Shapley values. They can be characterized by the same system of axioms, the only difference being the $\alpha$-marginality axiom employed. Therefore, they can be distinguished by the notion of $\alpha$-marginality.

Myerson (1977) suggests an extension of the Shapley value to games in partition function form. Nevertheless, his value is not an $\alpha$-Shapley value. The following example illustrates this fact.

Example 6 Let $v$ be game with the player set $\{1,2,3\}$, where

$$
\begin{array}{r}
v(\{2,3\},\{\{1\},\{2,3\}, \emptyset\})=1, \\
v(\{1,3\},\{\{2\},\{1,3\}, \emptyset\})=2, \\
v(\{1\},\{\{1\},\{2,3\}, \emptyset\})=4,
\end{array}
$$

and $v(S, \mathcal{P})=0$ otherwise. Then the Myerson value of the game $v$ is $\phi^{M}(v)=$ $\left(\frac{8}{3}, \frac{-11}{6}, \frac{-5}{6}\right)$. If $\phi$ is an $\alpha$-Shapley value, then $\phi(v)=\left(\frac{4 a}{3}, \frac{-4 a-3}{6}, \frac{-2 a+2 b+1}{6}\right)$, where $0 \leq$ $a, b \leq 1$ and $a+b=1$. We can see that $\phi(v) \neq \phi^{M}(v)$.

Besides the $\alpha$-Shapley values proposed by Fujinaka (2004), Macho-Stadler et al. (2007) propose another class of extensions of the Shapley value for games in partition function form in which each value is constructed through the "average approach". Let $a$ be a real-valued function defined on the set of all embedded coalitions. We assume that $\sum_{\mathcal{P} \in \Gamma, \mathcal{P} \ni S} a(S, \mathcal{P})=1$ for each $S \subseteq N$. Let $v$ be a game. The average approach consists of 2 stages. First, we construct a characteristic function form game $\tilde{v}$ associated with the game $v$ and the function $a$ by assigning to each coalition $S \subseteq N$ the worth

$$
\tilde{v}(S)=\sum_{\mathcal{P} \in \Gamma, \mathcal{P} \ni S} a(S, \mathcal{P}) v(S, \mathcal{P})
$$

Macho-Stadler et al. (2007) refer to $a(S, \mathcal{P})$ as the "weight" of the partition $\mathcal{P}$ in the computation of the worth of $S \in \mathcal{P}$. Second, the average approach produces a value $\phi$ for games in partition function form by defining $\phi(v)=S h(\tilde{v})$ for all games $v$. We 
say a value is constructed through the average approach if it can be derived in the two stages procedure described above.

Macho-Stadler et al. (2007) show that their value $\varphi^{*}$ can be constructed through the average approach and the Bolger and Myerson values are not in their class. The externality-free value and our value $\Phi$ can be constructed through the average approach. Albizuri et al. (2005) also propose a value in this class.

Let $S \subseteq N$ and $S \neq \emptyset$. Denote $\Pi_{S}$ to be the set of all permutations $\pi$ of $N$ such that $\pi S=S$. Given a non-trivial embedded coalition $(S, \mathcal{P})$, a permutation $\pi \in \Pi_{S}$ and a game $v$, the game $\pi_{S, \mathcal{P}} v$ is defined by

$$
\left(\pi_{S, \mathcal{P}} v\right)(T, \mathcal{R})= \begin{cases}v(S, \pi \mathcal{P}), & \text { if }(T, \mathcal{R})=(S, \mathcal{P}), \\ v(S, \mathcal{P}), & \text { if }(T, \mathcal{R})=(S, \pi \mathcal{P}), \\ v(T, \mathcal{R}) & \text { o.w. }\end{cases}
$$

Let $v$ be a game and $\pi \in \Pi$. The game $\pi v$ is defined by $\pi v(S, \mathcal{P})=v(\pi S, \pi \mathcal{P})$ for all embedded coalitions $(S, \mathcal{P})$. We say that a value $\phi$ satisfies the strong symmetry axiom if

(1) for any permutation $\pi$ of $N, \phi(\pi v)=\pi \phi(v)$,

(2) for any non-trivial embedded coalition $(S, \mathcal{P})$ and for any permutation $\pi \in \Pi_{S}$, $\phi\left(\pi_{S, \mathcal{P}} v\right)=\phi(v)$.

We say that the weights used in the average approach are symmetric if they only depend on the size distribution of the partition. Macho-Stadler et al. (2007) propose the strong symmetry axiom and obtain the following result.

Theorem 7 (Macho-Stadler et al. 2007) Assume that the value $\varphi$ satisfies the weak dummy property and linearity (a strong version of additivity), Then $\varphi$ can be constructed through the average approach if and only if it satisfies the strong symmetry axiom. Furthermore, the weights used in the average approach must be symmetric and satisfy the following condition:

$$
a(\mathcal{P}(i), \mathcal{P})=\sum_{S \in \mathcal{P}, S \neq \mathcal{P}(i)} a\left(\mathcal{P}(i) \backslash i, \gamma_{i}(S, \mathcal{P})\right)
$$

for all $i \in N, \mathcal{P} \in \Gamma$ with $|\mathcal{P}(i)|>1$.

It is quite interesting to discuss the values constructed through the average approach with the positive and symmetric weights satisfying (9).

Theorem 8 If a value is constructed through the average approach with the positive and symmetric weight function satisfying (9), then it is an $\alpha$-Shapley value.

Proof Let $\phi$ be the value constructed through the average approach with the positive and symmetric weight function $a$ satisfying (9). Let $v$ be a game and let $i \in N$. Then

$$
\phi_{i}(v)=\sum_{T \subseteq N, i \in T} \frac{(|T \backslash i| !)(|N \backslash T| !)}{|N| !}(\tilde{v}(T)-\tilde{v}(T \backslash i))
$$

where $\tilde{v}$ is defined by (8). Let $i \in N$ and let $\mathcal{P} \in \Gamma$. If $|\mathcal{P}(i)|>1$, then $a(\mathcal{P}(i), \mathcal{P})>0$. Let 


$$
\alpha_{i}\left(\mathcal{P}(i) \backslash i, \gamma_{i}(S, \mathcal{P})\right)=\frac{a\left(\mathcal{P}(i) \backslash i, \gamma_{i}(S, \mathcal{P})\right)}{a(\mathcal{P}(i), \mathcal{P})}
$$

for all $S \in \mathcal{P}$ and $S \neq \mathcal{P}(i)$. If $\mathcal{P}(i)=\{i\}$, let

$$
\alpha_{i}\left(\mathcal{P}(i) \backslash i, \gamma_{i}(S, \mathcal{P})\right)=\frac{1}{|\mathcal{P}|-1}
$$

for all $S \in \mathcal{P}$ and $S \neq \mathcal{P}(i)$.

For $T \subseteq N$ with $i \in T$ and $|T|>1$, it holds that

$$
\{\mathcal{O} \in \Gamma: \mathcal{O} \ni T \backslash i\}=\bigcup_{\mathcal{P} \in \Gamma, \mathcal{P} \ni T}\left\{\gamma_{i}(S, \mathcal{P}): S \in \mathcal{P}, S \neq \mathcal{P}(i)\right\}
$$

and

$$
\begin{aligned}
\tilde{v}(T)-\tilde{v}(T \backslash i)= & \sum_{\mathcal{P} \in \Gamma, \mathcal{P} \ni T} a(T, \mathcal{P}) v(T, \mathcal{P})-\sum_{\mathcal{O} \in \Gamma, \mathcal{O} \ni T \backslash i} a(T \backslash i, \mathcal{O}) v(T \backslash i, \mathcal{O}) \\
= & \sum_{\mathcal{P} \in \Gamma, \mathcal{P} \ni T}\left[\sum_{S \in \mathcal{P}, S \neq \mathcal{P}(i)} a\left(\mathcal{P}(i) \backslash i, \gamma_{i}(S, \mathcal{P})\right)\right] v(\mathcal{P}(i), \mathcal{P}) \\
& -\sum_{\mathcal{P} \in \Gamma, \mathcal{P} \ni T} \sum_{S \in \mathcal{P}, S \neq \mathcal{P}(i)} a\left(\mathcal{P}(i) \backslash i, \gamma_{i}(S, \mathcal{P})\right) v\left(\mathcal{P}(i) \backslash i, \gamma_{i}(S, \mathcal{P})\right) \\
= & \sum_{\mathcal{P} \in \Gamma, \mathcal{P} \ni T} \sum_{S \in \mathcal{P}, S \neq \mathcal{P}(i)} a\left(\mathcal{P}(i) \backslash i, \gamma_{i}(S, \mathcal{P})\right) \\
& \times\left[v(\mathcal{P}(i), \mathcal{P})-v\left(\mathcal{P}(i) \backslash i, \gamma_{i}(S, \mathcal{P})\right)\right] \\
= & \sum_{\mathcal{P} \in \Gamma, \mathcal{P} \ni T} \sum_{S \in \mathcal{P}, S \neq \mathcal{P}(i)} a(\mathcal{P}(i), \mathcal{P}) \alpha_{i}\left(\mathcal{P}(i) \backslash i, \gamma_{i}(S, \mathcal{P})\right) \\
& \times\left[v(\mathcal{P}(i), \mathcal{P})-v\left(\mathcal{P}(i) \backslash i, \gamma_{i}(S, \mathcal{P})\right)\right] \\
= & \sum_{\mathcal{P} \in \Gamma, \mathcal{P} \ni T} a(\mathcal{P}(i), \mathcal{P}) v_{\alpha}^{i}(\mathcal{P}) .
\end{aligned}
$$

If $|T|=1$, it follows that $v(T \backslash i, \mathcal{O})=0$ for all $\mathcal{O} \in \Gamma, \mathcal{O} \ni T \backslash i$ and

$$
\begin{aligned}
\tilde{v}(T)-\tilde{v}(T \backslash i) & =\sum_{\mathcal{P} \in \Gamma, \mathcal{P} \ni T} a(T, \mathcal{P}) v(T, \mathcal{P}) \\
& =\sum_{\mathcal{P} \in \Gamma, \mathcal{P} \ni T} a(\mathcal{P}(i), \mathcal{P})\left[\sum_{S \in \mathcal{P}, S \neq \mathcal{P}(i)} \frac{1}{|\mathcal{P}|-1}\right] v(\mathcal{P}(i), \mathcal{P}) \\
& =\sum_{\mathcal{P} \in \Gamma, \mathcal{P} \ni T} a(\mathcal{P}(i), \mathcal{P}) v_{\alpha}^{i}(\mathcal{P}) .
\end{aligned}
$$

We derive that $\phi$ satisfies the $\alpha$-marginality property. Since $\phi$ also satisfies efficiency and symmetry, it is the $\alpha$-Shapley value. 


\section{References}

Albizuri MJ, Arin J, Rubio J (2005) An axiom system for a value for games in partition function form. Int Game Theory Rev 7:63-73

Bolger EM (1989) A set of axioms for a value for partition function games. Int J Game Theory 18:37-44 de Clippel G, Serrano R (2008) Marginal contributions and externalities in the value. Econometrica 76:1413-1436

Dutta B, Ehlers L, Kar A (2008) Externalities, potential, value and consistency. Working Paper, Department of Economics, University of Warwick, UK

Fujinaka Y (2004) On the marginality principle in partition function form games. Mimeo, Graduate School of Economics, Kobe University, Japan

Macho-Stadler I, Pérez-Castrillo D, Wettstein D (2007) Sharing the surplus: an extension of the Shapley value for environments with externalities. J Econ Theory 135:339-356

McQuillin B (2009) The extended and generalized Shapley value: simultaneous consideration of coalitional externalities and coalitional structure. J Econ Theory 144:696-721

Myerson RB (1977) Values of games in partition function form. Int J Game Theory 6:23-31

Pham Do KH, Norde H (2007) The Shapley value for partition function form games. Int Game Theory Rev 9:353-360

Potter A (2000) A value for partition function form games. Working Paper, Department of Mathematics, Hardin-Simmons University Abilene, Texas, USA

Shapley LS (1953) A value for $n$-Person games. In: Kuhn H, Tucker A (eds) Contributions to the theory of games II. Princeton University Press, Princeton, pp 307-317

Thrall RM, Lucas WF (1963) $n$-Person games in partition function form. Naval Res Logist Quart 10:281298

Young HP (1985) Monotonic solutions of cooperative games. Int J Game Theory 14:65-72 